\title{
Optimisation of Additively Manufactured Permanent Magnets for Wind Turbine Generators
}

\author{
Connor McGarry \\ Electronic \& Electrical Engineering \\ University of Strathclyde \\ Glasgow, Scotland \\ connor.mcgarry@strath.ac.uk
}

\author{
Alasdair McDonald \\ Electronic \& Electrical Engineering \\ University of Strathclyde \\ Glasgow, Scotland \\ alasdair.mcdonald@strath.ac.uk
}

\author{
Nasser Alotaibi \\ Electronic \& Electrical Engineering \\ University of Strathclyde \\ Glasgow, Scotland \\ nasser.alotaibi.2017@strath.ac.uk
}

\begin{abstract}
With the increased demand for higher efficiency electrical machines, renewable energy and in all-electric transport systems, there is a growing market for permanent magnet machines and hence usage of rare earth magnet materials. One application - direct drive wind turbines - has a particularly large requirement in terms of magnet $\mathrm{kg} / \mathrm{MW}$ and an aspiration to reduce this usage. That in turn motivates the authors of this paper to examine the optimal shape, distribution and mixture of permanent magnet poles by that could be produced through an additive manufacturing (AM) route. $A M$ is a relatively new technique of magnet manufacture which has the potential to replace conventional forming techniques that are wasteful and often struggle with complex geometries. A genetic algorithm coupled to a finite element code is used to optimise magnet size and material configuration, and compares the results with conventional manufacturing/shaping techniques. This proposal investigates if additive manufacturing can meet the same level of performance whilst reducing permanent magnet material cost. Results which use additive manufacturing show that a reduction in the total magnet cost can be achieved with virtually no penalties in overall machine performance. Although the evaluation focuses on the cost of the rare earth magnet material in use - and excludes manufacturing cost and time - the results highlight that by using additive manufacturing a cost reduction of up to $3 \%$ can be achieved highlighting that AM has significant potential to compete with, if not succeed, existing magnet forming techniques.
\end{abstract}

Index Terms-additive manufacturing, permanent magnets, permanent magnet generators, direct-drive wind turbines.

\section{INTRODUCTION}

$\mathbf{T}$ HROUGH the desire for higher efficiency electric motors and an increased use in all-electric transport and renewable energy applications, the demand for permanent magnet machines is increasing. The use of Permanent Magnet Generators (PMGs) in wind turbine technology has proved to be an effective solution to many of the challenges faced by conventional generators [1] [2]. In addition to the requirement of regular maintenance, the high failure rates associated with the use of gearboxes has driven the advancement of directdrive, gearless systems [3] [4]. The generators used for these turbines have a significant impact on the overall performance, which, in turn, affects the energy output and subsequently the ability to meet the reduction targets. Unfortunately, the low rotational speed means that the torque rating of these wind turbine generators can be massive. The torque and the mass of magnet material are closely linked, and PMGs in direct drive wind turbines use very large quantities of rare earth magnet materials. The use of these rare earth magnets, specifically Neodymium Iron Boron (NdFeB), contributes significantly to the overall cost of these machines. The price volatility of this material can also be a significant risk to generator and turbine manufacturers. There is an appetite to find ways of reducing both mass and cost of this magnet material. This paper looks to combine two strategies that have been proposed by others to minimise total magnet use and cost. The first strategy (as proposed by [5] and others) looks to use additive manufacturing (AM) techniques producing near-net shape magnets, avoiding the usual wastage in magnet forming. The second strategy (as proposed by [6] and others) uses a non-homogeneous mix of magnet materials and grades in magnetic pole. This is normally done with 3 or more distinct magnet blocks - formed conventionally - out of two or more magnet grades. The two approaches are combined here. To evaluate this, a genetic algorithm in MATLAB was coupled to a magnetostatic finite element modelling software code (FEMM [7]) to evaluate non-homogenous additively manufactured magnetic poles. The results obtained allow for a cost analysis providing insight into the optimal design of magnets - from a perspective of magnet material costs - while meeting the flux density of the conventionally formed magnets techniques. As a case study, the authors chose a baseline machine design from Polinder $e t$ al.'s paper, 'Comparison of Direct-Drive and Geared Generator Concepts for Wind Turbines' [8]. This paper presents both the rotor and stator design of a $3 \mathrm{MW}$ PMG for a direct drive wind turbine. In this case, the modelling maintains the same stator and airgap dimensions and allows the magnet to vary. The same magnetic loading (as defined by the fundamental flux density) is conserved so that the machine will have similar losses and electrical characteristics.

\section{BACKGROUND}

\section{A. Permanent Magnet Machine Design}

A PM machine refers to any machine whereby the rotor field excitation is generated through the use of permanent magnets [2]. These machines are typically very robust, lightweight, have a small size and offer high efficiencies (because of the 
lack of Joule losses on the rotor). The use of permanent magnets has been seen in a variety of different machines. In wind turbines there have been various configurations used in the design of PMGs, reflecting the development in magnet properties and the increasing demand for improved efficiencies [2]. There are three broad families of rotating PMGs: radial-, axial, and transverse-flux machines, indicating the direction that the flux crosses airgap between the rotor to stator. Underneath these families there are further sub-groups which describe the rotor design, magnet mounting and degree of saliency (e.g. surface-mounted, buried-magnet, flux-concentrating, interior PM machines) [3]. There are also variations of stator design which effect the resulting airgap flux density, examples include iron-cored/slotted machines, slotless/airgap windings and aircored/coreless machines. This paper uses a radial-flux surfacemounted PMG to study partly because it is the most basic machine type and partly because it was used originally in [8]. The approach could be readily applied in other PMGs if required.

\section{B. Magnet Shaping}

The formation of magnetic materials and their properties is a complex science and is the domain of material scientists and chemists. A number of papers have looked at the impact of AM on magnet material properties (e.g. [5]). These papers identify that there is a significant difference in relation to the genetic structure of the magnets in contrast with conventional magnets primarily stemming from the material form required for the AM process. Conventionally, the process of magnet formation is well understood in comparison with AM therefore, conventional magnets are generally more advanced in comparison with AM magnets. As a result of this the authors are concerned more about the freedom in shaping the magnet and its impact on the available flux and, consequently, the overall machine efficiency, i.e. working at the machine level not microscopic level.

Wu et al. in [9] highlight various magnet shapes emphasising different designs as shown by Fig 1, that may be beneficial for different PM machine applications.

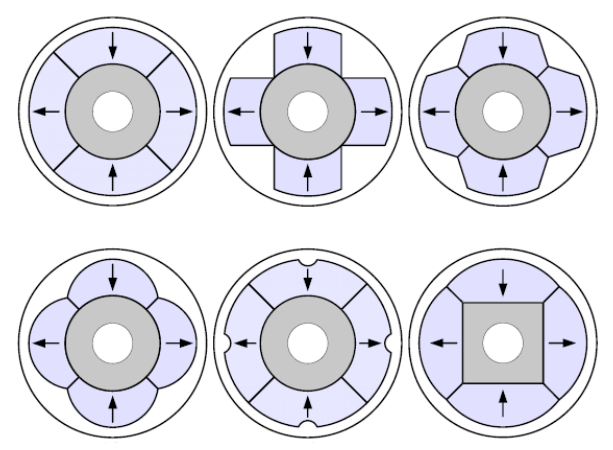

Fig. 1. Magnet shapes achievable through conventional techniques [9].

The highlighted designs, however, would result in excessive waste of permanent magnet material in manufacturing and would also significantly increase the manufacturing time and complexity if using conventional manufacturing techniques. Grinding, drilling, and Electric Discharge Machining (EDM) are examples of techniques used currently within industry to machine and shape magnets. Working on a relatively brittle, temperature-sensitive material, limits the magnet shapes that are available by using these manufacturing techniques and tends to limit the design space for the machine designer.

\section{Additive Manufacturing (AM)}

AM, commonly known as 3D printing, has been used for rapid prototyping and predominately for plastic components [10]. However, with technological advancement, metals and ceramics are becoming more commonly used. The process of AM involves, in most cases, using a computer rendered design to produce a component layer by layer [10]. In relation to permanent magnets, different techniques have been developed to achieve this, for example; Fused Deposition Modelling (FDM), Big Area Additive Manufacturing (BAAM), Selective Laser Melting (SLM) and Electron Beam Melting (EBM) [10] [11].

AM provides the ability to manufacture complex geometries composed of different materials whilst minimising wastage. Additionally, in using AM the overall complexity of a given design has little impact on the cost of manufacture compared with conventional techniques [5]. AM also has the potential to add features that, for example, improve magnet cooling by integrating cooling channels allowing lower cheaper grades to be used [5]. These attributes are highly desirable in comparison with conventional techniques which are typically wasteful and limited in terms of shape manufacturing. AM in principle is not necessarily a new technology, having been researched and developed for more than 20 years [12]. As previously mentioned briefly with technological advancement, the development of AM for metals and ceramics is increasing. Reference [10] provides an in-depth overview of AM techniques, providing cost analysis and case studies of different techniques briefly touching upon AM of permanent magnets. Reference [11] focuses specifically on magnet AM, primarily on bulk material properties and magnetic structure. Application of different techniques are not extensively covered therefore making it difficult to determine an optimum technique for specific use, such as a PMG. Due to the current state of $\mathrm{AM}$, in terms of material properties still being significantly behind material properties of conventional techniques, the vast majority of literature focuses predominately on the magnetic structure of materials. As a result of this, there is not one specific AM technique used in the development of this paper, as previously alluded to, there are several techniques which are still in the very early stages of producing AM magnets which would potentially be capable of achieving the magnet composites and geometries seen later in the paper. Literature such as [13] provides an overview of existing AM techniques as a whole. As manufacturing permanent magnets through AM is still a significantly new concept, the full potential of this technique in relation to PMGs is not yet fully understood, providing suitable grounds for this paper. 


\section{Generator MODEL}

This section of the paper provides an overview of the model and the associated properties whilst emphasising the assumptions and simplifications used to reduce the complexity of the model.

\section{A. Dimensions}

Fig 2 represents the model outlining key dimensions, these dimensions are defined within Table I. The dimensions outlined within Table I are fixed throughout the modelling process.

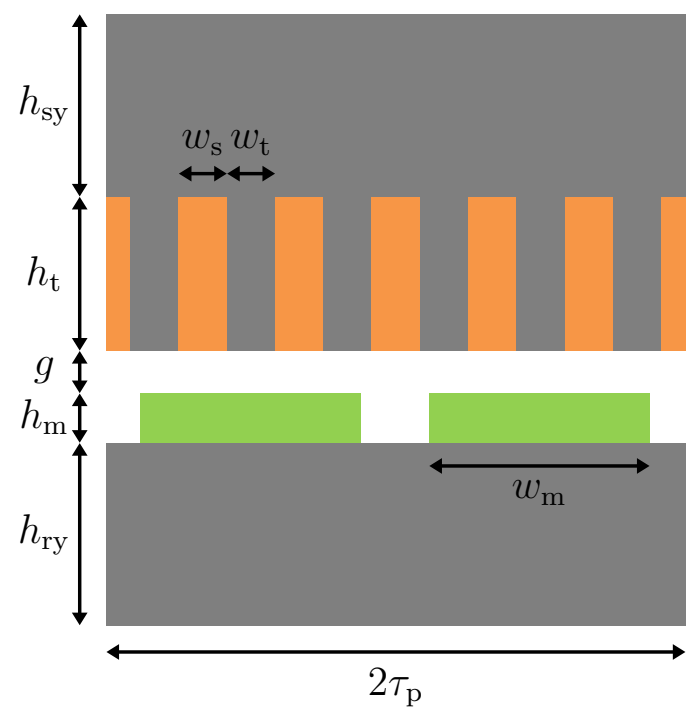

Fig. 2. Representation of the model highlighting key dimensions. Permanent Magnet M-36 Steel Copper Wire.

TABLE I

Generator Model Machine Dimensions

\begin{tabular}{lc}
\hline \hline Machine Dimensions & $\mathrm{mm}$ \\
\hline Rotor Yoke Height $\left(h_{\mathrm{ry}}\right)$ & 40 \\
Stator Yoke Height $\left(h_{\mathrm{sy}}\right)$ & 40 \\
Teeth Height $\left(h_{\mathrm{t}}\right)$ & 80 \\
Slot Height $\left(h_{\mathrm{s}}\right)$ & 80 \\
Airgap $(g)$ & 5 \\
Tooth Width $\left(w_{\mathrm{t}}\right)$ & 18 \\
Slot Width $\left(w_{\mathrm{s}}\right)$ & 15 \\
Pole Pitch $\left(\tau_{\mathrm{p}}\right)$ & 99 \\
Machine Length $(l)$ & 1200 \\
\hline
\end{tabular}

The magnet height $\left(h_{\mathrm{m}}\right)$ and width $\left(w_{\mathrm{m}}\right)$ are variable dimensions and are constrained as boundary conditions throughout the optimisation process.

\section{B. Materials}

The materials used and their properties are highlighted within this section of the paper. Table II highlights the properties of the fixed materials for the model with Table III highlighting the magnet material properties.
TABLE II

Model Material Properties

\begin{tabular}{ccccccc}
\hline \hline Material & $\begin{array}{c}\mu \\
\left(\mathrm{H} / \mathrm{m}^{2}\right)\end{array}$ & $\begin{array}{c}\text { Cduct } \\
(\mathrm{MS} / \mathrm{m})\end{array}$ & $\begin{array}{c}\text { Lam Thick } \\
(\mathrm{mm})\end{array}$ & Lam Fill & $\begin{array}{c}\text { Density } \\
\left(\mathrm{kg} / \mathrm{m}^{3}\right)\end{array}$ & $\begin{array}{c}\text { Cost } \\
(€ / \mathrm{kg})\end{array}$ \\
\hline $\begin{array}{c}\text { Air } \\
\text { Copper }\end{array}$ & 1 & - & - & - & 1.225 & - \\
M-36 Steel & 1616 & 58 & - & - & 8960 & 15 \\
\hline \hline
\end{tabular}

TABLE III

MAGNet PROPERTIES

\begin{tabular}{lcccccc}
\hline \hline Material & $\begin{array}{c}\mathrm{HcB} \\
(\mathrm{kA} / \mathrm{m})\end{array}$ & $\begin{array}{c}\mu \\
\left(\mathrm{H} / \mathrm{m}^{2}\right)\end{array}$ & $\begin{array}{c}\mathrm{Br} \\
(\mathrm{T})\end{array}$ & $\begin{array}{c}\text { Cduct } \\
(\mathrm{MS} / \mathrm{m})\end{array}$ & $\begin{array}{c}\text { Density } \\
\left(\mathrm{kg} / \mathrm{m}^{3}\right)\end{array}$ & $\begin{array}{c}\text { Cost } \\
(€ / \mathrm{kg})\end{array}$ \\
\hline $\mathrm{N} 35 \mathrm{H}$ & 770 & 1.12 & 1.08 & 0.667 & 7500 & 53 \\
$\mathrm{~N} 40 \mathrm{H}$ & 830 & 1.10 & 1.14 & 0.667 & 7500 & 60 \\
$\mathrm{~N} 45 \mathrm{H}$ & 880 & 1.08 & 1.19 & 0.667 & 7500 & 69 \\
$\mathrm{~N} 50 \mathrm{H}$ & 955 & 1.05 & 1.25 & 0.667 & 7500 & 77 \\
\hline
\end{tabular}

In relation to Air, Copper and M-36 Steel the data set stems from an original material library within FEMM with minor adjustments. The varying PM grade properties are obtained from analysing the $\mathrm{BH}$ curves for each grade.

\section{Assumptions and Simplifications}

Traditionally magnets are curved in order to fit the geometry of the rotor however in terms of modelling this proves to be an overly complex process therefore, used by McDonald in [14] for simplicity, the magnet shape can be considered as a cube. In doing so the FEMM model is simplified significantly improving simulation run time without having a detrimental impact on the accuracy of the results. To further improve the simplicity and simulation run time the use of boundaries is considered. By using periodic boundaries, the machine can essentially be sectioned minimising the machines complexity, subsequently, reducing the model size therefore further improving simulation run time. By linearising arcs and using linear BH curves further simplification can be achieved.

\section{Methodology}

As the paper seeks to determine the potential of AM as a magnet manufacturing technique compared with conventional techniques, it is important to remark upon the relative magnet quality of the state of the art. As is the case with sintered and bonded magnets, there are significant differences in maximum energy product $B H_{\max }$ between sintered magnets and those that have been produced by AM. Given that the magnetic properties associated with a magnet manufactured through AM are significantly lower than the properties achieved through conventional manufacturing techniques, in order to make viable comparisons, Fig 3 is considered. AM magnets have achieved $B H_{\max }$ in the range of 5-18 MGOe [15], whereas a designer can source relatively economical sintered magnets in the range of 40-50 MGOe. In this paper it is assumed that with time AM magnet material will achieve the same $B H_{\max }$ as conventional magnets, this assumption is represented by 
Fig 3. Future studies have scope to investigate which level of $B H_{\max }$ produced by $\mathrm{AM}$ would be competitive relative to the conventional magnet.

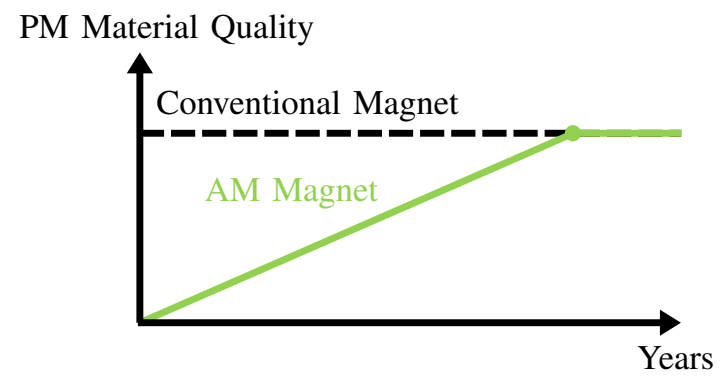

Fig. 3. Design assumption relating to conventional magnet material quality compared with AM magnet quality.

Based on this initial assumption, optimisation and comparison of the five scenarios outlined by Fig 4 represents the primary focus of the paper. By investigating these different scenarios, the full scale of AM's capabilities in relation to PMG design potential is explored; crucially, determining if, by utilisation of AM, the same efficiency can be achieved at a reduced cost. The five scenarios are compared based on the objective function of magnet mass minimisation, under a constraint of delivering at least the baseline minimum magnetic loading (expressed as the fundamental airgap flux density). Fig 4a represents a conventional magnet and using the machine dimensions outlined within Polinder's paper, the focus of this scenario relates solely to the optimisation of magnet width and height. Fig 4b highlights the introduction of AM with the primary focus relating specifically to the optimisation of magnet width, height and material configuration which is essentially the assignment of material per region, using two different magnet grades. Here the size and shape of these regions is fixed. This simplifies the optimisation as it reduces the number of independent variables. Similarly, to Fig $4 \mathrm{~b}$, Fig $4 \mathrm{c}$ follows the same process, however, introduces a third magnet grade to investigate if there is an impact on overall magnet cost. Fig 4d and Fig 4e essentially follows the same process however, the size of regions is reduced, increasing the number of regions. The approaches in Fig $3 \mathrm{~b}-\mathrm{e}-$ which choose between discrete materials for each region - lend themselves to a Genetic Algorithm (GA) optimisation.

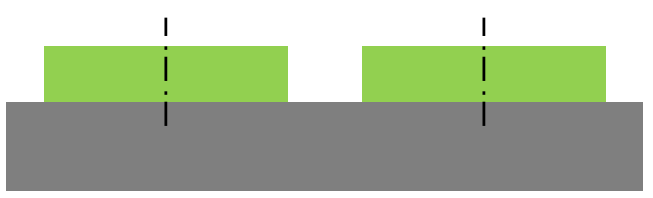

(a) Conventional Magnet - 1 material.

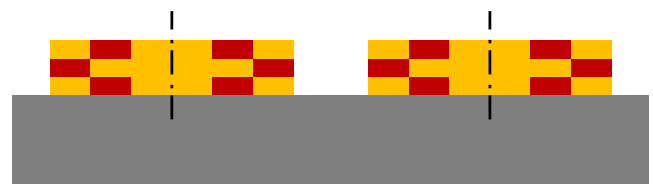

(b) AM magnet - 2 materials.

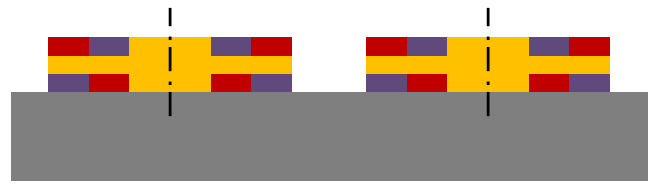

(c) AM magnet - 3 materials.

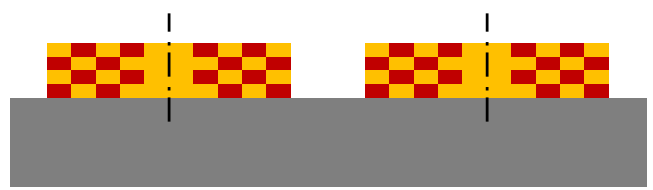

(d) AM magnet - 2 materials with reduced domain size.

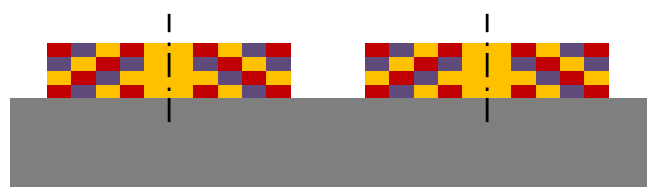

(e) AM magnet - 3 materials with reduced domain size.

Fig. 4. Optimisation Scenarios.

$\mathrm{N} 50 \mathrm{H}$

$\mathrm{N} 45 \mathrm{H}$

$\mathrm{N} 40 \mathrm{H}$ $\mathrm{N} 35 \mathrm{H}$

Fig 5 highlights the optimisation strategy, the figure identifies that the magnet is essentially split into two sections with the first section being the primary focus of the optimiser, the second section is a direct mirror of the first section as shown. This magnet is then duplicated whilst switching the polarity for the second pole. This technique simplifies the optimisation process and reduces the computational run time.

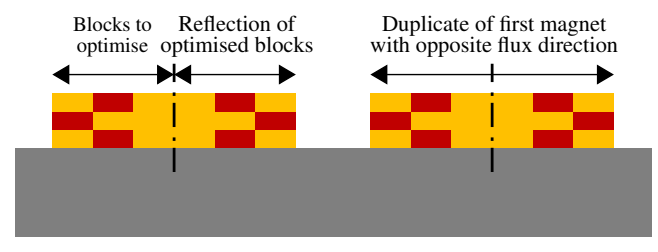

Fig. 5. Optimisation strategy.

Each material is given a numerical value and the material configuration matrix is constructed based on the number of columns and rows used to form the magnet. The example shown represents the material configuration demonstrated by Fig 5. By continuously changing the positioning of the numerical values within the matrix this allows for the authors to investigate the impact differing material configurations have on the overall magnet cost and efficiency.

$$
\text { Material Configuration }=\left[\begin{array}{lll}
1 & 0 & 1 \\
0 & 1 & 1 \\
1 & 0 & 1
\end{array}\right]
$$

Fig 6 highlights the basic optimisation process used to determine the optimum design. The optimisation in MATLAB changes the independent variables, produces a LUA file with appropriate dimensions and materials which is passed through to the finite element software, FEMM, generating a linearised 2D model. This executes a magnetostatic run and extracts airgap flux density waveform. This data is then analysed harmonically to determine the airgap flux density in MATLAB. 
The magnet mass is also calculated in MATLAB. In following this process, FEMM can be fully utilised entirely through MATLAB.

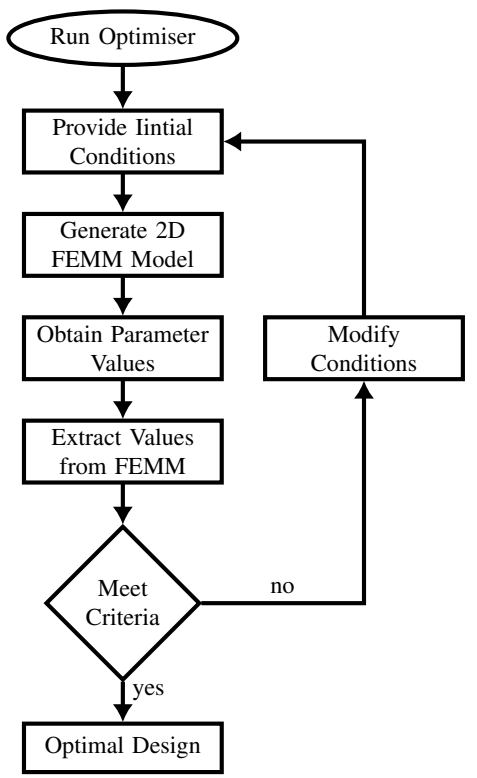

Fig. 6. Optimisation process utilising both FEMM and MATLAB.

In order to effectively investigate the cost of AM magnets in comparison with conventional magnets the harmonic impact associated with the change in magnet geometry must be considered. The flux density in the teeth, $B_{\mathrm{t}}$ and the flux density in the stator, $B_{\mathrm{s}}$ are calculated through utilising the calculation process outlined by equations (1) - (7).

$$
\begin{aligned}
& A_{\mathrm{g}}=w_{\mathrm{m}} \times l \\
& A_{\mathrm{t}}=3 w_{\mathrm{t}} \times l \\
& A_{\mathrm{s}}=h_{\mathrm{sy}} \times l \\
& \frac{A_{\mathrm{t}}}{A_{\mathrm{g}}}=\frac{3 w_{\mathrm{t}}}{w_{\mathrm{m}}} \\
& \frac{A_{\mathrm{s}}}{A_{\mathrm{g}}}=\frac{h_{\mathrm{sy}}}{w_{\mathrm{m}}} \\
& B_{\mathrm{t}}=\frac{\phi_{\mathrm{g}}}{A_{\mathrm{t}}}=\frac{\phi_{\mathrm{g}}}{\frac{3 w_{\mathrm{t}}}{w_{\mathrm{m}}} A_{\mathrm{g}}}=\left(\frac{w_{\mathrm{m}}}{3 w_{\mathrm{t}}}\right) \times B_{\mathrm{g}} \\
& B_{\mathrm{s}}=\frac{\frac{1}{2} \phi_{\mathrm{g}}}{A_{\mathrm{g}}}=\frac{\frac{1}{2} \phi_{\mathrm{g}}}{\frac{h_{\mathrm{sy}}}{w_{\mathrm{m}}} A_{\mathrm{g}}}=\left(\frac{w_{\mathrm{m}}}{2 h_{\mathrm{sy}}}\right) \times B_{\mathrm{g}}
\end{aligned}
$$

where $A_{\mathrm{g}}$ represents the area of the airgap, $A_{\mathrm{t}}$ the area of the teeth, $A_{\mathrm{s}}$ the area of the stator, $\phi_{\mathrm{g}}$ the flux in the airgap and $B_{\mathrm{g}}$ the flux density in the airgap.

The teeth and stator losses are then calculated based on the Weibull distribution and iron loss equation highlighted within
Polinder's paper with an adaptation, represented by (8). The adaptation involved removing the factor of 2 used to approximate iron losses as this study requires direct calculation of the losses associated with each individual harmonic to assess the impact of the differing magnet manufacturing techniques.

$$
P_{\mathrm{Fe}}=P_{\mathrm{Fe} 0 \mathrm{~h}}\left(\frac{f_{\mathrm{e}}}{f_{0}}\right)\left(\frac{\hat{B}_{\mathrm{Fe}}}{\hat{B}_{0}}\right)^{2}+P_{\mathrm{Fe} 0 \mathrm{e}}\left(\frac{f_{\mathrm{e}}}{f_{0}}\right)^{2}\left(\frac{\hat{B}_{\mathrm{Fe}}}{\hat{B}_{0}}\right)^{2}
$$

where $B_{0}$ is the flux density, $f_{0}$ is the angular frequency, $f_{\mathrm{e}}$ is the frequency of the field in the iron, $P_{\mathrm{Fe} 0 \mathrm{~h}}$ is the given hysteresis loss per unit mass at the given $f_{0}$ and $B_{0}, P_{\mathrm{Fe} 0 \mathrm{e}}$ is the eddy current loss per unit mass at the given $f_{0}$ and $B_{0}$.

The total associated iron losses are calculated based on equation (9).

$$
P_{\mathrm{T}}=\sum_{n=1} P_{\mathrm{Fe}}\left(B_{\mathrm{t}, \mathrm{n}}, f_{\mathrm{n}}\right)+\sum_{n=1} P_{\mathrm{Fe}}\left(B_{\mathrm{s}, \mathrm{n}}, f_{\mathrm{n}}\right)
$$

where $P_{\mathrm{T}}$ is the total iron losses and $n$ is the harmonic number.

As AM has still a way to go in terms of magnet manufacturing the authors developed a 'Magnet Toolbox' that allows for continuous research of this developing technology in relation to PMG's. The MATLAB application is highlighted by Fig 7 emphasising the input quantities. Fig 7 represents the initial start-up screen, providing the user with the ability to run a variety of different simulations. Most importantly however, in relation to this paper the Toolbox allows for the authors to efficiently compare and contrast the different scenarios as previously highlighted. The toolbox consists of multiple tabs providing a variety of different functions such as ability to change material properties and cost as well as view and extract results from any simulations.

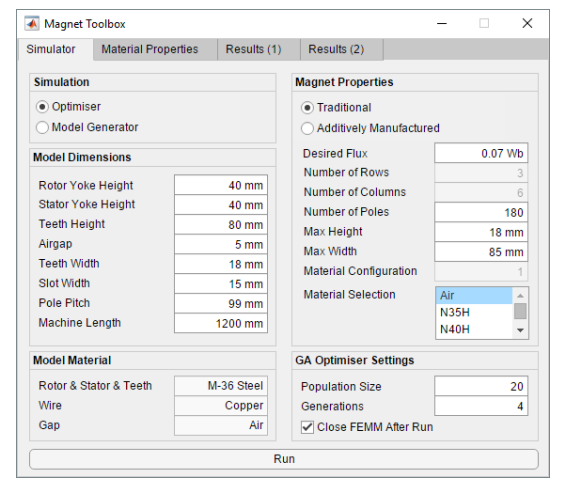

Fig. 7. Magnet Toolbox input variables for model generation or optimisation.

\section{RESULTS}

The results obtained from running the optimisation scenarios are highlighted within this section of the paper. Primarily focusing on the comparison between the AM magnets and the conventional magnet however, the effect resulting from changing the number of materials as well as the domain size is also considered. Fig 8 highlights the magnet topology capable of achieving a flux of $0.07 \mathrm{~Wb}$ for each scenario. 

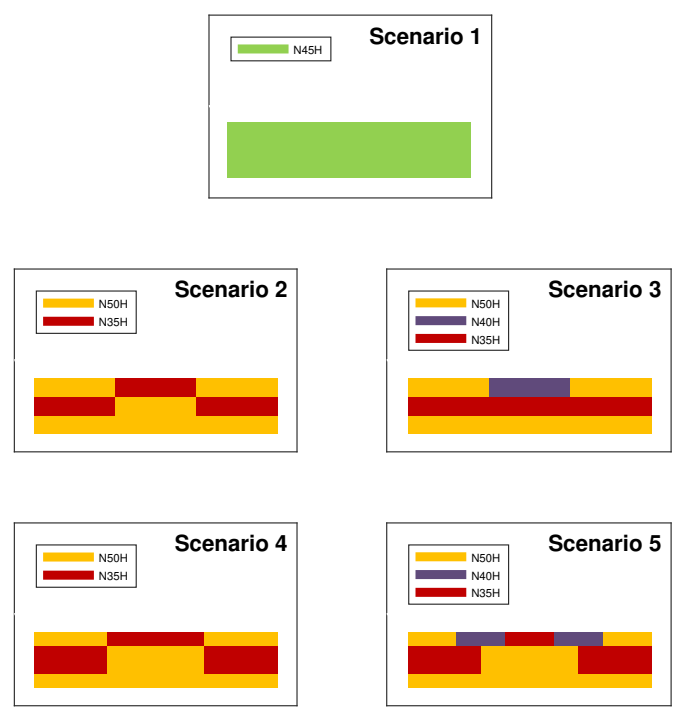

Fig. 8. Magnet configurations.

Based on the magnet configurations obtained from the optimiser Fig 9 highlights the FEMM model for each scenario emphasising the behaviour of the flux in each case.

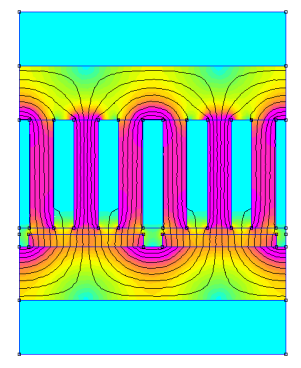

(a) Scenario 1

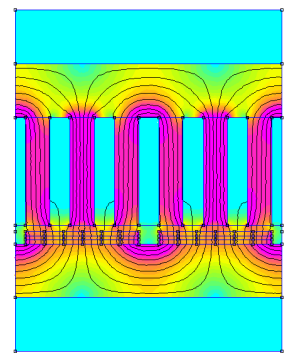

(b) Scenario 2

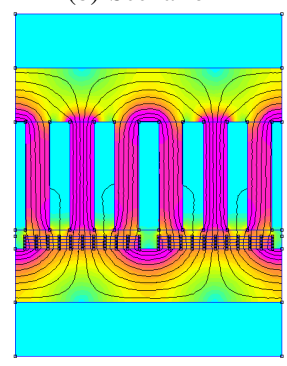

(d) Scenario 4

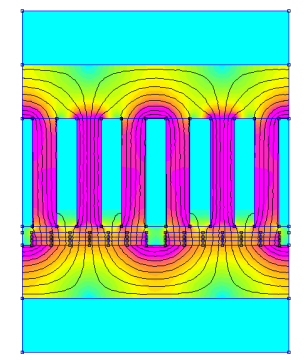

(c) Scenario 3

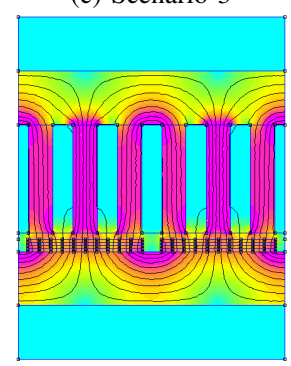

(e) Scenario 5
Fig. 9. FEMM model for each of the differing scenarios.
In all cases the optimiser found a magnet height of $9 \mathrm{~mm}$ with a width of $85 \mathrm{~mm}$ except for scenario 2 where the width was $84 \mathrm{~mm}$. As a result the airgap flux density waveforms are relatively similar despite the change in magnet configuration although there are subtle differences which are likely to have a minor impact on the harmonic losses associated with each scenario.
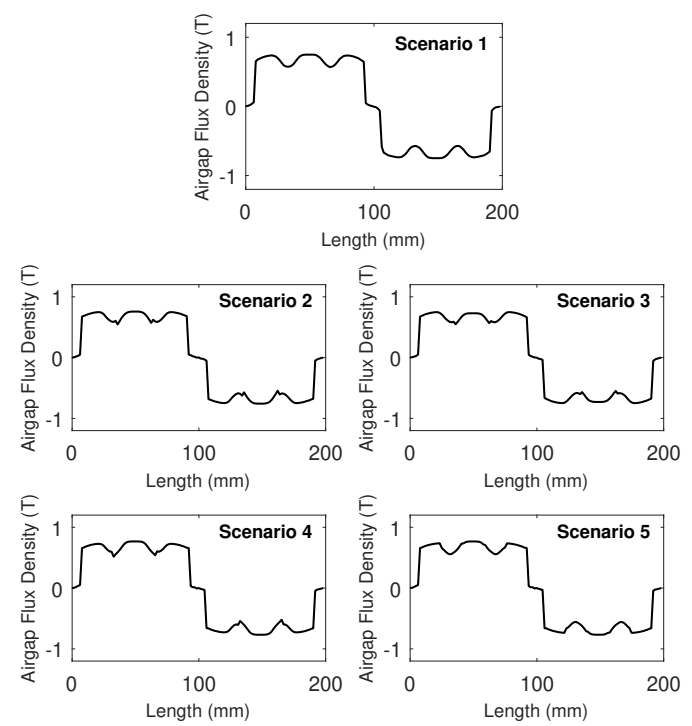

Fig. 10. Comparison of the airgap flux density waveform for each scenario.

Fig 11 highlights the harmonic content within the airgap for each of the scenarios emphasising again similarity but with subtle differences.
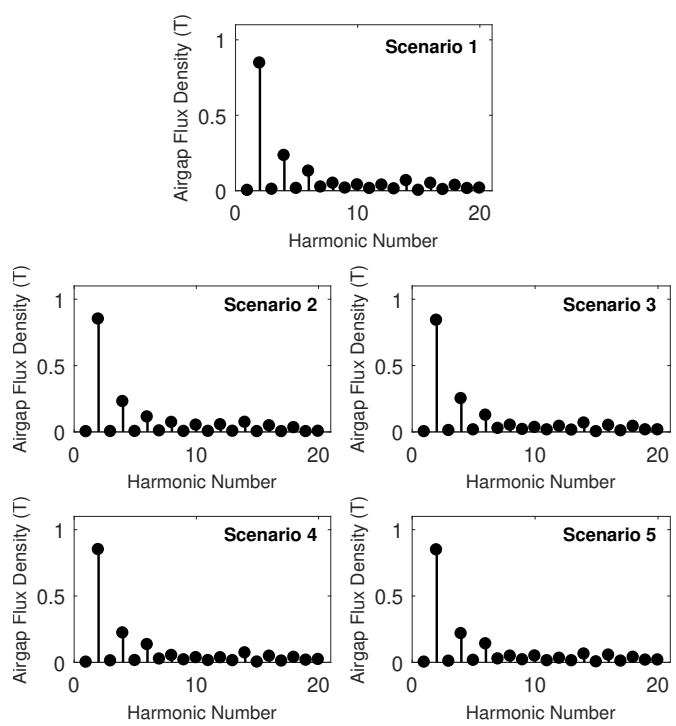

Fig. 11. Comparison of the airgap flux density spatial harmonics for each scenario.

Fig 12 identifies cost savings in terms of percentage for scenarios $2-5$ in comparison with scenario 1 . The figure highlights savings ranging from $1.18 \%-3.13 \%$. The figure 
additionally highlights a trend in relation to the behaviour of the AM scenarios in that the scenarios which utilise three differing magnets grades result in a greater saving than in comparison with the scenarios that use two magnet grades. Further savings are also seen when the domain size is reduced, comparing scenarios 2 and 4 as well as 3 and 5 the scenario with smaller domain size resulted in a greater saving for both cases.

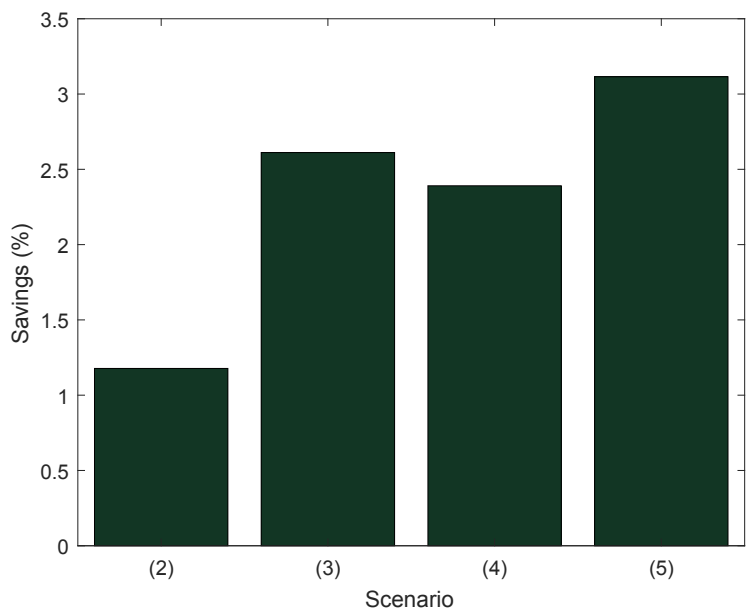

Fig. 12. Percentage saving of material cost for scenarios 2-5 in comparison with scenario 1 .

Despite having a direct saving on material cost, the impact of changing the magnet structure on the overall machine losses must be considered to fully assess the cost effectiveness of AM magnets in comparison with conventional magnets. The iron losses of the generator in $\mathrm{kW} / \mathrm{kg} / \mathrm{yr}$ are highlighted by Fig 13 for each of the scenarios.

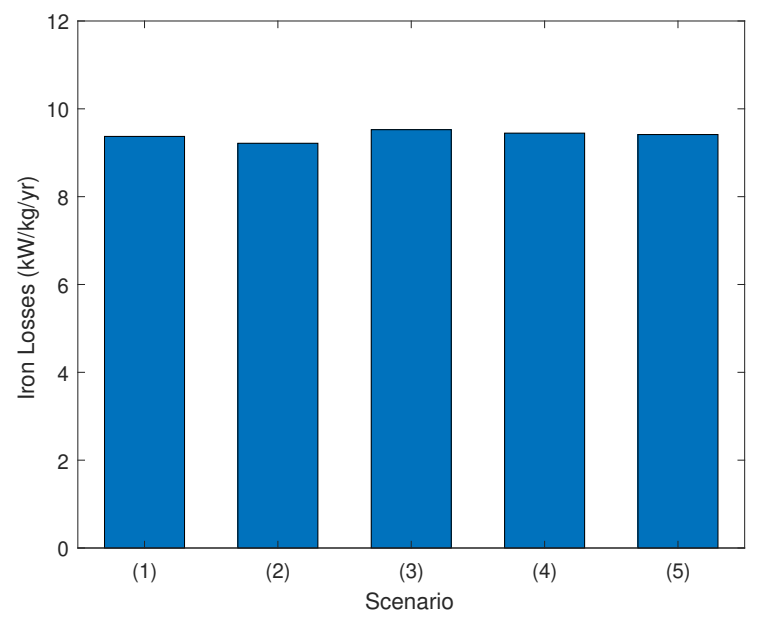

Fig. 13. Iron losses for the machine per year for each scenario.

The figure emphasises that the iron losses are relatively similar which is expected as seen by previous figures. Reduction in losses seen at scenario 2 is suspected to be a result of having smaller dimensions in comparison with the other scenarios. Such a minor difference is unlikely to have a significant detrimental impact on the cost therefore identifying that by using AM magnets similar overall machine characteristics can be achieved at a reduced material cost.

\section{DISCUSSIONS}

The study conducted provides a first look into the potential of AM magnets as means of replacing conventional magnets. The findings indicate that from a purely cost perspective AM has the ability to provide a reduction in total material cost in comparison with conventional techniques without seeing any significant penalty in machine performance. In relation to optimisation difficultly arose in relation to computational run time of each simulation which ultimately limited the scope of the paper. Equation (10) highlights the calculation used to determine the number of solutions $N$, where $N_{\mathrm{M}}$ is the number of materials in use, $N_{\mathrm{R}}$ is the number of rows and $N_{\mathrm{C}}$ is the total number of columns. Table IV highlights the number of potential solutions for each scenario for a given height and width.

$$
N=N_{\mathrm{M}}^{\left(N_{\mathrm{R}} \times \frac{N_{\mathrm{C}}}{2}\right)}-1
$$

TABLE IV

Number of Potential Solutions Per Magnet Height And Width

\begin{tabular}{ccccc}
\hline \hline Scenario & $N_{\mathrm{M}}$ & $N_{\mathrm{R}}$ & $N_{\mathrm{C}}$ & Solutions \\
\hline 1 & 1 & - & - & 1 \\
2 & 2 & 3 & 6 & 511 \\
3 & 3 & 3 & 6 & 19,682 \\
4 & 2 & 4 & 10 & $1,048,575$ \\
5 & 3 & 4 & 10 & $3,486,784,400$ \\
\hline \hline
\end{tabular}

This ultimately emphasises the difficultly in determining the global optimum for the AM scenarios due to the significantly large search space whilst considering that the magnet height and width are also being optimised within bounds of $9 \mathrm{~mm}$ - $18 \mathrm{~mm}$ for the height and $80 \mathrm{~mm}-85 \mathrm{~mm}$ for the width. The optimiser did find results with savings and similarities however, it is statistically likely that it missed combinations with lower magnet costs. As no mathematical or analytical model exists yet this made the optimisation process significantly challenging. Furthermore throughout the testing process findings indicate that the material configuration had virtually no impact on the flux itself meaning that optimising based on a material configurations ability to achieve a given flux in relation to cost was not necessarily applicable in comparison with the amount of each material per configuration for example which would impact both cost and flux.

Findings highlight that by introducing a greater number of magnet grades into the mix, material cost savings can be obtained whilst achieving the same flux, in principle this is achieved by utilising a material with a high magnet grade in conjunction with a lower magnet grade. The findings also 
highlight that as the domain size is reduced greater savings can be made without any impact on the flux achieved. However, as shown the savings are minimal and although solutions may have been missed it has to be recognised that by using this technique, with the magnet grades presented, it is unlikely to result in substantial savings in relation to magnet cost in use. This does not necessarily hold true for all possible applications of $\mathrm{AM}$ in terms of magnet manufacture as further research into the use of unconstrained domain sizes and shapes coupled with an improved optimisation approach i.e. analytical could potentially increase savings.

A crucial factor to consider is that by using different magnet grades for a static model of this nature the optimiser essentially provides the most cost efficient configuration and dimensions based on the position of the magnet in relation to the teeth. This however in a realistic environment may not necessarily be applicable as the magnet moves from the fixed position during rotation. As different magnet grades are located throughout the magnet when the rotor moves the contribution from different areas of the magnet essentially changes and based on the position in relation to the teeth the ability to provide a constant contribution as with the conventional magnet is challenged. Further investigation would ultimately be required to assess this in terms of a full model capable of simulating different rotor positions, how this impacts cogging torque for example must also be investigated.

\section{CONCLUSION}

To conclude, this paper has presented an introduction to the potential of AM magnets for PMGs. The paper aimed to investigate if AM magnets could result in a cheaper overall magnet material cost in comparison with conventional magnets, with findings emphasising that a reduction of upto $3 \%$ can be achieved. The challenges associated with the optimisation approach taken in this paper are emphasised however, given the amount of potential solutions and findings, the importance of developing an analytical model for future research also needs to be acknowledged. The paper concludes that AM magnets have potential to compete with conventional magnets specifically in relation to material cost however, for a full cost comparison manufacturing cost and time would have to be taken into consideration. Further investigation into the impact on key machine characteristics is required to assess all ramifications associated with this change in magnet manufacturing technology. An assumption relating to the availability of high quality AM magnets allowed for this study however, future studies should consider the possibility that this may not necessarily be the case and through AM only limited magnet properties can be achieved.

\section{REFERENCES}

[1] A. Grauers, "Efficiency of three wind energy generator systems," IEEE Transactions on Energy Conversion, vol. 11, no. 3, pp. 650-657, 1996.

[2] V. Akhmatov, "Modelling and ride-through capability of variable speed wind turbines with permanent magnet generators," Wind Energy, vol. 9, no. 4, pp. 313-326, 2006.
[3] S. Eriksson, H. Bernhoff, and M. Leijon, "Evaluation of different turbine concepts for wind power," Renewable and Sustainable Energy Reviews, vol. 12, no. 5, pp. 1419-1434, 2008.

[4] N. Madani, "Design of a permanent magnet synchronous generator for a vertical axis wind turbine," 2011

[5] L. Li, A. Tirado, Nlebedim et al., "Big area additive manufacturing of high performance bonded ndfeb magnets," Scientific Reports, vol. 6, p. $36212,2016$.

[6] A. H. Isfahani, S. Vaez-Zadeh, and M. A. Rahman, "Using modular poles for shape optimization of flux density distribution in permanentmagnet machines," IEEE Transactions on Magnetics, vol. 44, no. 8, pp. 2009-2015, Aug 2008.

[7] D. Mekeer, "Finite element method magnetics." [Online]. Available: http://www.femm.info/wiki/HomePage

[8] H. Polinder, F. F. Van der Pijl, G.-J. De Vilder, and P. J. Tavner, "Comparison of direct-drive and geared generator concepts for wind turbines," IEEE Transactions on energy conversion, vol. 21, no. 3, pp. 725-733, 2006.

[9] L. Wu and Z. Zhu, "Analytical modeling of surface-mounted pm machines accounting for magnet shaping and varied magnet property distribution," IEEE Transactions on Magnetics, vol. 50, no. 7, pp. 1-11, July 2014.

[10] M. Mann, Palmer et al., "The current state of additive manufacturing in wind energy systems," Oak Ridge National Laboratory (ORNL), Tech. Rep., 2017.

[11] V. Popov, A. Koptyug, I. Radulov, F. Maccari, and G. Muller, "Prospects of additive manufacturing of rare-earth and non-rare-earth permanent magnets," Procedia Manufacturing, vol. 21, pp. 100 - 108, 2018, 15th Global Conference on Sustainable Manufacturing.

[12] N. Guo and M. C. Leu, "Additive manufacturing: technology, applications and research needs," Frontiers of Mechanical Engineering, vol. 8, no. 3, pp. 215-243, 2013.

[13] F. Calignano, D. Manfredi, E. P. Ambrosio, S. Biamino, M. Lombardi, E. Atzeni, A. Salmi, P. Minetola, L. Iuliano, and P. Fino, "Overview on additive manufacturing technologies," Proceedings of the IEEE, vol. 105, no. 4, pp. 593-612, 2017.

[14] A. McDonald and N. A. Bhuiyan, "On the optimization of generators for offshore direct drive wind turbines," IEEE Transactions on Energy Conversion, vol. 32, no. 1, pp. 348-358, 2017.

[15] M. P. Paranthaman, I. C. Nlebedim et al., "Additive manufacturing of permanent magnets," Lawrence Livermore National Laboratory, Tech. Rep., 2016. 